\title{
ALGUNAS CLAVES PARA UNA PSICOTERAPIA DE ORIENTACIONN FEMINISTA EN MUJERES QUE HAN PADECIDO VIOLENCIA DE GÉNERO
}

\author{
ESPERANZA BOSCH FIOL*, VICTORIA A. FERRER PÉREZ* Y AINA ALZAMORA** \\ * Grupo de investigación "Estudios de género». Universidad de las Islas Baleares ${ }^{1}$ \\ ** Psiquiatra. Práctica privada. Palma de Mallorca.
}

\section{INTRODUCCIÓN}

En este trabajo vamos a desgranar cuales deben ser, en nuestra opinión, los elementos esenciales para desarrollar una psicoterapia de orientación feminista en la atención a las mujeres (y sus hijos/as) que han padecido violencia de género y, particularmente, violencia en la pareja.

En este sentido, cabe recordar que hoy en día la consideración más asumida es que la violencia contra las mujeres es un fenómeno multicausal que sólo puede ser explicado a partir de la intervención de un conjunto de elementos diversos, incluyendo factores individuales, sociales y del contexto concreto ${ }^{2}$.

Pero, incluso si hablamos de múltiples causas, en la base de esta pirámide causal encontraríamos una concepción sexista de los agresores, estrechamente ligada a la exacerbación del modelo masculino tradicional (que incluye la consideración de lo masculino como superior, la legitimidad de la supeditación femenina al varón,...) y una estructura social económica y familiar patriarcal que implica una distribución desigual del poder que impregna la construcción social del género y de la sexualidad y que afecta profundamente a las relaciones entre los hombres y las mujeres ${ }^{3}$.

1. Dirección de contacto: Facultad de Psicología. Universidad de las Islas Baleares. Ctra. Valldemossa, km 7'5. 07122 Palma de Mallorca. Baleares. Tel. 971-17-34-40. Fax 971-17-31-90. E-mail: esperanza.bosch@uib.es

2. HEISE, Lori L.: «La violencia contra la mujer. Organización global para el cambio», en Jeffrey L. Edleson y Zvi C. Eisikovits (eds.): Violencia doméstica: La mujer golpeada y la familia, Barcelona, Granica, 1997, pp. 19-58. BOSCH, Esperanza y FerRER, Victoria A.: La voz de las invisibles. Las víctimas de un mal amor que mata, Madrid, Ed. Cátedra. Col. Feminismos, 2002.

3. CORSI, Jorge: "Masculinidad y violencia», en Jorge Corsi: Violencia masculina en la pareja. Una aproximación al diagnóstico y a los modelos de intervención, Buenos Aires, Paidós, 1995, pp. 27-40. 
La denuncia del sistema patriarcal, que impone una jerarquización violenta tanto en el seno del grupo familiar como, por extensión, en la sociedad en su conjunto, se ha convertido en el eje central del análisis del fenómeno de la violencia de género. En un trabajo anterior ${ }^{4}$ recordábamos las diversas declaraciones de organismos internacionales en los que se insiste en la necesidad de la prevención y tratamiento de este tipo de violencia, y la necesidad de la consideración del mismo como un grave problema social.

Así, la Asamblea General de Naciones Unidas adoptó la "Declaración sobre la eliminación de la violencia contra la mujer» de $1994^{5}$ que constituye el primer instrumento internacional de derechos humanos que aborda de forma explícita la violencia de género y la define como "todo acto de violencia basado en el género que tiene como resultado posible o real un daño físico, sexual o psicológico, incluidas las amenazas, la coerción o la privación arbitraria de la libertad, ya sea que ocurra en la vida pública o en la vida privada».

Desde 1995 y dentro del programa de desarrollo y salud de la mujer, la Organización Mundial de la Salud ha dedicado esfuerzos a este tema. Desde este programa se desarrollan y coordinan los trabajos sobre violencia contra las mujeres que inicialmente se centraron en violencia doméstica y luego se han diversificado hacia otros ámbitos (conflictos armados...). En este marco, en febrero de 1996 se acordó considerar la definición de violencia contra las mujeres adoptada por Naciones Unidas como un marco útil para las actividades de la OMS. A mediados de ese año se estableció un grupo especial sobre violencia y salud para coordinar las diversas actividades sobre este tema. En mayo de ese mismo año, la 49a Asamblea Mundial de la Salud adoptó una resolución (WHA 49.25) constatando el aumento notable de la incidencia de lesiones intencionales que afectaban a personas de todas las edades y de ambos sexos, pero especialmente a mujeres y niños; reconociendo las graves consecuencias inmediatas y a largo plazo que, para el desarrollo psicológico y social de los individuos, las familias, las comunidades y los países, tiene la violencia; declarando la violencia como prioridad de salud pública; e instando a sus Estados Miembros a evaluar el problema y a tomar medidas para prevenirlo y resolverlo.

Al año siguiente, la OMS adoptó una nueva resolución (WHA 50.19) incidiendo en la importancia de reconocer la violencia contra las mujeres como un problema social y sanitario de primera magnitud y que requiere acción urgente.

GERBER, G.: "Gender stereotypes and the problem of marital violence», en L. Adler y F. Denmark (eds.): Violence and the prevention of violence, New York, Praeger, 1995. HeISE, Lori L.: Op. cit. LOERENTE, Miguel: Mi marido me pega lo normal, Barcelona, Crítica, 2001.

4. BosCH, Esperanza; Ferrer, Victoria A.; AlZAMORA, Aina y NAVARRO, C.: «Itinerarios hacia la libertad. La recuperación integral de las víctimas de la violencia de género», Psicología y Salud, 15:1 (2005), pp. 97-105.

5. ORGANIZACIÓN DE NACIONES UNIDAS (ONU): Declaración sobre la eliminación de la violencia contra las mujeres (Res. A/R/48/104), Nueva York, Naciones Unidas, 1994. 
Algunas claves para una psicoterapia de orientación feminista en mujeres que...

Según esta organización ${ }^{6}$, las razones que convierten a la violencia contra las mujeres en un tema prioritario para los/as profesionales de la salud son las siguientes: a) En primer lugar, y aunque su verdadera extensión sea difícil de conocer, pues en muchas ocasiones es un "delito oculto", la violencia produce padecimiento y consecuencias negativas para aproximadamente un $20 \%$ o más de la población femenina; b) En segundo lugar, y a pesar de que su carácter de "delito oculto» hace en ocasiones difícil establecer exactamente cuáles son sus consecuencias, cada vez son más los trabajos que señalan el impacto negativo de la violencia contra las mujeres sobre importantes temas de salud (maternidad sin riesgo, planificación familiar, enfermedades crónicas, etc.); c) Y, en tercer lugar, para muchas mujeres víctimas los/as profesionales de la salud son su punto de apoyo más importante.

Con todo ello se trataba de que los estados miembros y también las instituciones y los/as profesionales que se ocupan directamente del tema tomaran plena conciencia de la gravedad del problema y de la necesidad de arbitrar mecanismos para la imprescindible prevención y atención a las mujeres afectadas, y en muchos casos también a sus hijos/as.

Partiendo de la aceptación de que estas premisas, según las cuales la violencia de género se nutre de los presupuestos patriarcales $y$, por tanto, de una clara asimetría entre hombres y mujeres, el proceso de superación de una mujer víctima tiene que ir más allá del simple cambio de conductas o de estrategias individuales de mejora, para alcanzar la comprensión de los mecanismos sociales y culturales que han actuado en la génesis del problema. Quizás de esta manera se pueda evitar algo que también actúa como un prejuicio en contra de ellas: la repetición de relaciones basadas en el abuso.

En este marco, el tratamiento psicoterapéutico, tanto individual como en grupo, se convierte en una herramienta básica para la recuperación integral de la víctima. Sin embargo si quien lo administra no tiene la formación necesaria, o parte de presupuestos basados exclusivamente en las características internas de la mujer afectada (como ha sido propio de la psicología y psiquiatría clínica tradicional), la efectividad del mismo puede quedar seriamente comprometida.

Así, como señala Romero ${ }^{7}$, a la hora de hablar de las intervenciones psicológicas para la atención a las mujeres maltratadas son muchos los aspectos en los que se podría profundizar, incluyendo, entre otros, los modelos psicoterapéuticos de elección, su eficacia, las características idóneas de los/as terapeutas, la inclusión y el manejo de la perspectiva de género en los tratamientos,

6. ORGANIZACIÓN MUNDIAL DE LA SALUD (OMS): Violencia contra la mujer, http://www.who. int/frh-whd/VAW/infopack/Spanish/violencia-infopack.htm, 1998. ORGANIZACIÓN MUNDIAL DE LA SALUD (OMS): Gender and health a technical paper, http://www.who.int/frh-whd/ GandH/Ghreport/gendertech.htm, 1998. ORGANIZACIÓN MUNDIAL DE LA SALUD (OMS): Violence and injury prevention. Violence and health, http://www.who.int/eha/pvi/infokit/women. htm, 1999.

7. ROMERO, Inmaculada: «Desvelar la violencia: una intervención para la prevención y el cambio», Papeles del Psicólogo, 88 (2004), pp. 19-25. 
la jerarquización de los propios objetivos de tratamiento, los criterios de éxito o la importancia del trauma, entre otras cuestiones. De algunas de ellas vamos hablar en las páginas siguientes.

\section{LA MIRADA TRADICIONAL MÉDICO-PSICOLÓGICA}

A finales de siglo XIX la psicología avanza ya con paso firme como una disciplina independizada del tronco filosófico y con pretensiones claramente experimentales. Desde esta nueva disciplina científica, o con pretensiones de serlo, se plantea el estudio del comportamiento humano, tanto individual como en grupo, de una manera rigurosa y objetiva, mediante métodos fiables e intentando marcar distancias con posturas más especulativas. Pero, a pesar del rigor metodológico que demuestra en otros aspectos, en lo que respecta a las mujeres apenas diferencia entre tradición y verdad científica. Así, muchas de las creencias ancestrales sobre su inferioridad intelectual y biológica, sobre su sexualidad y también sobre su psicopatología, traspasaron las puertas de esta nueva ciencia, prescindiendo de cualquier visión crítica ${ }^{8}$.

Curiosamente en la misma época, la mitad de este genero humano se organiza en la defensa de sus derechos como ciudadanas: su derecho al voto, a la educación, al trabajo remunerado, a su dignidad como persona, en definitiva. Pero la relación entre ambos, la nueva ciencia psicológica y los movimientos en defensa de los derechos de las mujeres, ni será fácil, ni dará frutos válidos hasta mucho más adelante 9 . Los más ancestrales prejuicios sobre la condición femenina y su transvase a la definición misma de los conceptos de masculinidad y feminidad de una manera rígida e inamovible, a los que se les atribuyen toda una serie de características y atributos, condicionarán, desde el mismo momento del nacimiento, la vida de las personas, marcando los límites de sus expectativas y aspiraciones, y señalando claramente cuáles van a ser las sanciones a las que se pueden ver expuestas si no se ciñen a ellas.

En este sentido, si repasamos las características atribuidas a hombres y mujeres, podremos comprobar como éstas tienen una muy distinta valoración social (siempre más favorables del lado de la masculinidad) y una jerarquización añadida según la cual la inferioridad de las mujeres en cuanto a su fragilidad, emotividad, pasividad etc, requiere de un varón protector y fuerte y seguro, que desde su «superioridad natural» sea el encargado de velar y controlar la vida de la mujer, sea la hermana, esposa, hija....

Vamos a resumir estas características atribuidas a la masculinidad y feminidad, para posteriormente compararlas con síntomas definitorios de determinados síndromes, como por ejemplo el depresivo.

8. BosCH, Esperanza; FerRer, Victoria A. y GILI, Margarita: Historia de la misoginia, Madrid, Antrophos, 1999.

9. BosCh, Esperanza; FerRer, Victoria A.; RIerA, Teresa y AlBerdi, Rosamaría: Feminismo en las aulas, Palma de Mallorca, Universitat de les Illes Balears, Colecció Treballs Feministes, 2003. 
Algunas claves para una psicoterapia de orientación feminista en mujeres que...

Así, por una parte, el trabajo de Williams y Benett sobre los contenidos de los estereotipos de género nos proporciona un ejemplo de cuáles son las características que habitualmente suelen aparecer en uno y otro de estos estereotipos ${ }^{10}$ :

\begin{tabular}{|l|l|}
\hline Estereotipo femenino tradicional & Estereotipo masculino tradicional \\
\hline Afectada & Agresivo \\
\hline Atractiva & Ambicioso \\
\hline Complaciente & Asertivo \\
\hline Coqueta & Austero \\
\hline De corazón blando & Auto-confiado \\
\hline Débil & Autocrático \\
\hline Dependiente & Aventurero \\
\hline Dócil & Con coraje \\
\hline Emocional & Cruel \\
\hline Encantadora & Desordenado \\
\hline Estirada & Digno de confianza \\
\hline Excitable & Dominante \\
\hline Femenina & Emprendedor \\
\hline Frívola & Estable \\
\hline Gentil & Excitable \\
\hline Habladora & Fuerte \\
\hline Llorona & Hábil \\
\hline Machacona & Independiente \\
\hline Remilgada & Lógico \\
\hline Sensible & Masculino \\
\hline Sentimental & No emotivo \\
\hline Sofisticada & Racional \\
\hline Soñadora & Realista \\
\hline Sumisa & Resistente \\
\hline Voluble & Robusto \\
\hline & Seguro \\
\hline & Severo \\
\hline
\end{tabular}

Por otra parte, tal y como señalan Mas, Tesoro y Sanz ${ }^{11}$, las diferencias cualitativas en la morbilidad psiquiátrica entre uno y otro sexo podrían estar relacionadas, precisamente, con los estereotipos de género tradicionales que lleva a hombres y mujeres a responder diferencialmente ante sentimientos o estímulos negativos. Así, en función de las características propias del estereotipo asignado para cada género se tendería a padecer un tipo u otro de trastorno. En este

10. Williams, J.E. y BenETT, S.M.: «The definition of sex stereotypes via the Adjective Check List», Sex Roles, 1 (1975), pp. 327-337.

11. MAS, Josefina; TESORO, Amalia y SANZ, Ana I.: «Influencia de los factores sociales en la psicopatología de la mujer», en J. Mas y A. Tesoro (coords.): Mujer y salud mental. Mitos y realidades, Madrid, Asociación Española de Neuropsiquiatría, 1993, pp. 133-163. 
sentido, se constata que las características incluidas en el estereotipo tradicional femenino tienen muchos puntos en común con cuadros psicopatológicos como el depresivo o los trastornos afectivos. En cambio, las características del estereotipo tradicional masculino son más adaptativas al mundo actual y, en todo caso, apuntan hacia trastornos de personalidad antisocial o similares.

\begin{tabular}{|l|l|}
\hline Depresión & Feminidad \\
\hline Dependencia & Dependencia \\
\hline Pasividad & Pasividad \\
\hline Falta de firmeza o asertividad & Falta de firmeza o asertividad \\
\hline Gran necesidad de apoyo afectivo & Gran necesidad de apoyo afectivo \\
\hline Baja autoestima e indefensión & $\begin{array}{l}\text { Como efecto, se desarrolla baja autoestima e } \\
\text { indefensión }\end{array}$ \\
\hline Incompetencia & Incompetencia \\
\hline
\end{tabular}

\begin{tabular}{|l|l|}
\hline Depresión & Masculinidad \\
\hline Pasividad & Actividad \\
\hline Dependencia & Autonomía \\
\hline Falta de firmeza o asertividad & Asertividad y agresividad \\
\hline Gran necesidad de apoyo afectivo & $\begin{array}{l}\text { Capacidad de aceptar riesgos y tomar } \\
\text { decisiones }\end{array}$ \\
\hline Baja autoestima e indefensión & $\begin{array}{l}\text { Como efecto se desarrolla alta autoestima y } \\
\text { seguridad }\end{array}$ \\
\hline Incompetencia & Competencia \\
\hline
\end{tabular}

Centrándonos en el tema de la violencia de género, será gracias a los movimientos de mujeres, tanto fuera (feminismo social) como dentro de las universidades (feminismo académico), que esta forma de violencia empezó a ser considerada no solo como una gravísima lacra para la convivencia democrática, sino también como un ineludible objeto de estudio ${ }^{12}$. El proceso de comprensión de este fenómeno tampoco ha sido fácil ni ha estado exento de prejuicios.

Desde la psicología y la psiquiatría tradicional no siempre se han identificado las claves objetivas para entender el porqué de los comportamientos violentos de muchos hombres, ni la aparente pasividad y falta de reacción de las víctimas ${ }^{13}$. No obstante hoy en día estas claves están suficientemente definidas, de manera que ignorarlas no podrá ser considerado, en ningún caso, como inocente.

12. BosCH, Esperanza y FerReR, Victoria A.: «La violencia de género: De cuestión privada a problema social», Intervención Psicosocial. Revista de igualdad y calidad de vida, 9:1 (2000), pp. 7-19.

13. FERRER, Victoria A. y BosCH, Esperanza: "Introduciendo la perspectiva de género en la investigación psicológica sobre violencia de género", Revista Anales de Psicología, 21:1 (2005), pp. 1-10. Disponible en: http://www.um.es/analesps/v21/v21_1e.htm. 


\section{LA PSICOTERAPIA COMO PROCESO DE CAMBIO}

Si analizamos los grandes mitos femeninos, desde la mujer supuestamente masoquista hasta la devoradora de hombres, la frígida o la ninfómana, se nos muestra de una manera clara la doble moral con que se evalúa el comportamiento femenino y masculino, así como la evidencia de que todos estos hilos van tejiendo la tupida tela de araña, el laberinto, en el que viven muchas mujeres víctimas de violencia de género.

Como señala Sau, $\mathrm{V}^{14}$ en muchos casos, las psicoterapias tradicionales (a las que esta autora directamente denomina "psicoterapias patriarcales") no solo no han sabido dar respuestas adecuadas a las mujeres que han sufrido violencia de género, sino que han reforzado los mitos misóginos y, por tanto, condenado a la soledad y desamparo a mujeres que, paradójicamente, buscaban en la psicoterapia una solución y un consuelo. Si no se cuestiona el modelo social de base que condena a muchas mujeres a vivir sometidas a unos prejuicios que las convierten en "posesión afectiva» de sus compañeros y, por tanto, sometidas a ellos y a sus mandatos, cualquier otra actuación resultará, necesariamente, insuficiente.

En cualquier proceso psicoterapéutico el primer paso es saber atender y escuchar la demanda que se nos formula. Si quien escucha, parte de premisas sesgadas y de esquemas basados en prejuicios, el proceso se pervierte inmediatamente. Esta capacidad empática, tan necesaria para la práctica de la psicología en general, en el caso de las mujeres que han padecido violencia de género es absolutamente imprescindible.

Tratándose el problema de los malos tratos de una cuestión multifactorial $y$, generalmente, como ya se ha dicho, con consecuencias sobre el estado de salud, los equipos asistenciales (sanitarios, socio-sanitarios y de ayudas auxiliares del ámbito judicial) raramente dejan de intervenir en algún momento de la trayectoria de una historia de violencia. Serán, por tanto, varias las personas profesionales de la salud o asistenciales que tendrán acceso a la víctima y a su historia. De su capacidad de escucha activa, de sus conocimientos sobre el tema y de su compromiso dependerá en buena medida el éxito terapéutico, o, por el contrario, el cierre en falso del caso y el fracaso de todo el proceso.

Vamos a recordar brevemente los diferentes escenarios por los que puede transitar la petición de ayuda terapéutica de una mujer víctima de violencia de género.

\subsection{Centro de atención primaria}

\subsubsection{Atención primaria}

El primer escalón asistencial juega un papel determinante en la detección precoz de un problema de maltrato. Tanto por la especial relación de confianza

14. SAU, Victoria: «Psicología y feminismo(s)», en Ester Barberá e Isabel Martínez (coords.): Psicología y género, Madrid, Prentice Hall, pp. 107-118. 
que puede establecerse con el equipo de primaria, estimulada por la continuidad del mismo, como por ser el marco idóneo al que se puede acudir en búsqueda no explícita de auxilio.

La clínica que aportan las pacientes suele ser tórpida y, si no hay lesiones, enmascarada por la sintomatología psíquica en forma de astenia, trastornos del sueño y del apetito, manifestaciones temerosas de apariencia ansiosa, sintomatología somática sin correlato orgánico, etc. Cuadro que con el tiempo va derivando hacia otro de carácter depresivo, en el que con frecuencia se producen ideaciones e intentos de autolesión o suicidio.

Las lesiones, de cualquier tipo y localización se presentan, fundamentalmente, en cabeza y cuello, también son frecuentes en el tímpano y en zonas cubiertas por la ropa. En la mayoría de las ocasiones las pacientes dan explicaciones poco convincentes de como se han producido. Cuando estas lesiones son repetidas, van a más y existen daños de defensa, se debe establecer un diagnóstico de sospecha y poner en marcha los mecanismos asistenciales necesarios.

\subsubsection{Segundo nivel}

El deterioro psíquico se agudiza con la cronificación de este tipo de relación, la desesperanza y la dependencia bloquea su capacidad de elección y, por tanto, la resolución de su conflicto.

De esta manera se cierra el anillo emocional que, iniciándose con una relación amorosa, continuó con una reacción de negación, confusión, temor o shock ante la primera agresión, con un estado posterior de ansiedad por la previsión de la repetición de situaciones de violencia y por el estado depresivo con la depreciación por la vida y por si misma que enlaza con la cronificación señalada.

Cuando se inicia esta etapa, fundamentalmente si la paciente verbaliza deseos de morir y /o realiza algún intento de suicidio, suele ser derivada a la visita de especialidad. En este estadio es mas fácilmente detectable la presencia de malos tratos, por las manifestaciones de la mujer, no tanto sobre el hecho violento, que ella muchas veces no sólo no manifiesta sino que oculta (generalmente por considerarlo irrelevante e irremediable), como por las referencias que nos ofrece sobre su baja autoestima y a qué la refiere.

El tratamiento deberá involucrar a todo el equipo de salud, por diferentes motivos, algunos de los cuales conviene recordar brevemente:

a) Hacer frente a las posibles necesidades farmacológicas que puedan detectarse. En muchos casos serán los antidepresivos de alto umbral tóxico los más recetados, por lo que se debería hacer un seguimiento muy exhaustivo controlando en todo momento los posibles efectos secundarios y la excesiva sedación o tolerancia.

b) Necesidad de instaurar un tratamiento psicoterapéutico en la forma que se estime conveniente para cada paciente.

c) Por la información que ha de prestarse a las mujeres sobre los servicios comunitarios que puedan serle de ayuda a ellas y a sus hijos, sobre asociaciones 
Algunas claves para una psicoterapia de orientación feminista en mujeres que...

de mujeres o grupos de autoayuda que pueden ser cruciales en el proceso de su recuperación integral, o sobre posibles acciones legales.

\subsection{Servicios de Urgencia}

Las consultas a los servicios de urgencia no reflejan en muchas ocasiones la gravedad del problema de los malos tratos, debido a la vergüenza de las mujeres a dar explicaciones sobre lo ocurrido, al temor a las represalias del agresor y/o a las consecuencias familiares que pudieran derivarse de un parte clínico.

La actuación de estos servicios suele tener lugar ante lesiones graves en mujeres que aún están en condiciones de solicitar ayuda telefónica o que son halladas en este estado por familiares o vecinos que actúan como solicitantes. En estos casos la capacidad profesional y los recursos técnicos son tan importantes como la accesibilidad y empatía del personal sanitario.

\subsection{Servicios Sociales}

Junto a las intervenciones citadas que ocurren en los estadios de detección y tratamiento de las consecuencias del maltrato, los servicios sociales prestan servicios de información, apoyo y seguimiento, cubriendo sectores preventivos y de seguimiento, sin los cuales los servicios descritos podrían resultar muy poco efectivos.

La promoción y prevención en la salud estará vinculada a la red que los sectores político económicos, servicios sociales, usuarios y medios de información sean capaces de crear en una comunidad.

\subsection{Medicina Forense}

A este recurso se llega a petición judicial por denuncia interpuesta, por petición de investigación ante la sospecha de malos tratos o para estudio post mortem después del hallazgo de un cuerpo.

En las dos primeras situaciones, el equipo forense realizará la exploración física y psicodiagnóstica de la paciente y dará cuenta de los resultados al juzgado correspondiente.

Será de capital importancia en estos casos, recabar toda información relativa a su historia asistencial y clínica, exploración y resultados de pruebas complementarias diagnósticas y copia del informe que se hubiera confeccionado a efectos de ser requeridos para emisión de peritaje.

En todos los distintos eslabones de la cadena asistencial la preparación específica profesional será imprescindible.

\section{4. ¿QUÉ PRETENDE LA PSICOTERAPIA DE ORIENTACIÓN FEMINISTA?}

En nuestra opinión, la terapia de orientación feminista será aquella que, utilizando los instrumentos propios, parte de la evidencia de que la paciente es víctima no sólo de un sujeto violento, sino de una sociedad patriarcal que inculca la violencia como recurso legítimo del hombre para mantener el control sobre su pareja, a la que, a su vez, considera obligada a obediencia y sumisión. 
De manera que, en muchas ocasiones las mujeres tienen tan interiorizados los anti-valores patriarcales que vivirán con sentimientos de fracaso y culpabilidad su situación, tendiendo a justificar al agresor y a buscar en ellas mismas posibles explicaciones de las explosiones de ira de sus compañeros.

De acuerdo con ello, una primera característica que deberá tener esta psicoterapia será el estar basada en un modelo explicativo comprehensivo que permita una adecuada valoración y conocimiento de todos los factores individuales, sociales y del contexto que explican la violencia contra las mujeres, huyendo de explicaciones y planteamientos simplistas centrados exclusivamente en la psicología individual ${ }^{15}$.

Otro aspecto relevante y que se deriva de esa forma de entender el problema, es la necesidad de trabajar dentro de equipos multidisciplinares que puedan dar respuesta a las diferentes necesidades psicológicas, sanitarias, legales, laborales, de vivienda, etc. que puedan tener las mujeres y sus hijos e hijas, permitiendo una recuperación integral ${ }^{16}$.

Por lo que se refiere propiamente a las características de la intervención psicológica, un primer paso importante al iniciar la evaluación psicológica, es fijar con las mujeres el consentimiento informado ${ }^{17}$. Es decir, es importante explicar y lograr que comprendan por qué es relevante la evaluación, por qué hay que revisar ciertos temas, qué tipo de información se recogerá, cuál va a ser el uso que se le dará a toda esa información y quienes tendrán acceso a ella.

Al explicar su historia (bien sea porque se explica por primera vez, bien porque se revisan de nuevo acontecimientos sucedidos tiempo atrás...) las mujeres pueden reexperimentar el miedo, el dolor, la angustia, etc. que acompañaron a esos incidentes. Es importante, por ello, que la persona que ejerce de terapeuta sea sensible a esas emociones, facilite su expresión y canalización mientras recoge la información necesaria ${ }^{18}$.

Es fundamental, así mismo, que las mujeres «desnaturalicen»y "visibilicen» la violencia que han sufrido ${ }^{19}$. Son muchas las mujeres que han vivido relaciones abusivas sin ser conscientes de ello. Recordemos, por ejemplo, el concepto de "técnicamente maltratada» que se acuña en la macroencuesta del Instituto de la Mujer de 1999 para referirse a ellas ${ }^{20}$. Entender que todo»eso» que han vivido no es normal, no forma parte de las relaciones de pareja y no ha sido responsabilidad suya es el primer paso para superar su situación, para encontrar respuestas, para liberarse de los sentimientos de culpa y recuperar el control.

15. Goodman, Lisa A.; Koss, Mary P.; FitzGerald, Louise F.; Russo, Nancy F. y KeITA, Gwendolyn R.: "Male violence against women. Current research and future directions", American Psychologist, 48:10 (1993), pp. 1054-1058.

16. Matud, M. Pilar; Gutiérrez, Ana B. y PAdilla, Vanessa: «Intervención psicológica con mujeres maltratadas por su pareja», Papeles del Psicólogo, 88 (2004), pp. 1-9.

17. Ibid.

18. WALKER, Leonore E.A.: Abused women and survivor therapy: A practical guide for the psychotherapist, Washington DC, APA, 1994.

19. ROMERO, Inmaculada: Op. cit.

20. AlBerdi, Inés y MATAS, Nuria: Violencia doméstica, Barcelona, Fundación La Caixa, 2002. 
Algunas claves para una psicoterapia de orientación feminista en mujeres que...

Mary Ann Dutton ${ }^{21}$ propone un modelo para evaluar y planificar la intervención terapéutica en estos casos cuyas líneas maestras pueden ser de utilidad. Concretamente, esta autora propone que en la evaluación de las mujeres que han sufrido violencia a manos de sus parejas es necesario analizar las siguientes cuestiones:

a) El tipo y patrón de violencia, abuso y control ejercido por el agresor. El análisis del abuso es importante para comprender cuáles son o han sido sus efectos. Este análisis implica no sólo la descripción de los actos de violencia sino también la comprensión del contexto en el que ha ocurrido y el sentido que todo ello tiene para las mujeres. Esto es importante porque algunas conductas, aparentemente no violentas, (ciertas frases, ciertos tonos de voz, ciertos comportamientos...) pueden ser vividas por las mujeres con tanta o más ansiedad que la propia conducta violenta por haberse asociado a ésta repetidamente en el pasado.

b) Los efectos psicológicos de ese abuso. En este sentido es importante analizar tanto los indicadores de malestar psicológico (miedos, ansiedad, tristeza, consumo de sustancias....) como los cambios cognitivos (atribuciones, expectativas....) y las dificultades de relación con personas distintas del agresor (miedos, dificultad para establecer relaciones de intimidad...) etc. y, además, es importante partir de la base de considerar (al menos inicialmente) todas estas respuestas como consecuencias de las agresiones vividas. Ello permitirá superar uno de los prejuicios con los que en ocasiones se ha encarado el trabajo con las mujeres maltratadas y es el de considerar que la psicopatología que presentan era previa a la relación de abuso e, incluso, causa de ésta.

c) Las estrategias que las mujeres maltratadas han puesto en marcha para sobrevivir. En este sentido, es importante, recordar que las formas de afrontar las situaciones estresantes son muchas y diversas y no sólo las estrategias activas son afrontamiento. Así, es relativamente frecuente que las mujeres maltratadas que permanecen en la relación abusiva empleen estrategias pasivas y/o evitativas, lo cual ha sido en ocasiones interpretado erróneamente como "masoquismo», cuando en realidad no es más que una estrategia de supervivencia.

d) Los factores mediadores. Entre los que cabe destacar: la respuesta institucional, las creencias previas de las mujeres sobre la violencia, las relaciones de pareja y los roles de género, los recursos materiales y de apoyo social disponibles, la presencia de estresores adicionales o las experiencias previas. Todos estos factores, presentes de una u otra forma, pueden jugar tanto a favor (haciendo que las mujeres entiendan mejor la situación en la que se hallan y proporcionándoles recursos para salir de ella) como en contra (manteniendo a la mujer en la relación y aislada de posibles salidas) y deben ser valorados no sólo para conocer cuál es la situación de partida de cada mujer víctima en concreto,

21. DUTTON, Mary Ann: Empowering and healing the battered woman: a model of assessment an intervention, New York, Springer, 1992. 
sino también para saber cuáles son aquellos aspectos en los que va a ser necesario incidir principalmente a lo largo de la intervención posterior.

Por lo que se refiere a las técnicas de evaluación a emplear, lo habitual suele ser emplear entrevistas, tanto estructuradas como semiestructuradas que permiten obtener información sobre el abuso y sobre todas las áreas de la vida a las que acabamos de referirnos. A partir de ese uso común de las entrevistas se introducen posteriormente diferentes matices. Así, por ejemplo, Dutton ${ }^{22}$ considera importante realizar preguntas específicas y directas que permitan no olvidar ninguno de los aspectos relevantes a evaluar; Walter, L. ${ }^{23}$ considera necesario recoger la descripción de episodios de abuso concretos (como el primero, el más reciente o el peor) para conocer el contexto en el que se produce la violencia; Matad, y cols. ${ }^{24}$ proponen incluir una serie de cuestionarios para evaluar aspectos concretos (tales como la presencia de estrés postraumático, depresión, miedos...); etc. Según nuestra experiencia, una combinación de estas técnicas es efectiva siempre y cuando aseguren un clima de confianza con las mujeres y les permitan expresarse libremente.

Por lo que se refiere a propuestas de tratamiento, Lundy y Grossman ${ }^{25}$ han recogido y analizado hasta 16 posibles modelos de práctica clínica con mujeres maltratadas, valorando tanto los modelos empleados como los resultados obtenidos.

En nuestro entorno, entre los pioneros en el diseño y aplicación de programas de intervención para mujeres que han padecido violencia en la pareja están Enrique Echeburúa y cols. ${ }^{26}$. Estos autores proponen un tratamiento combinado que consta de 17 sesiones, 9 individuales y 8 grupales que se desarrollan en un período de 12 semanas y que abarcan la facilitación de la expresión de emociones, la reevaluación cognitiva de los sesgos presentados por las mujeres y la enseñanza de habilidades específicas de afrontamiento para abordar la situación de maltrato. El calendario de las sesiones se va adecuando en cada caso a las necesidades concretas que presentan las mujeres a las que se aplica.

Aunque la consideración de la violencia contra las mujeres en la pareja de la que parten estos autores es multicausal, incluyendo tanto factores individuales, como sociales y del contexto, el abordaje terapéutico de la violencia que proponen estaría, en nuestra opinión, más cercano a un planteamiento tradicional que a la perspectiva de género que venimos reclamando.

22. DutTon, Mary Ann: Op. cit.

23. WALKER, Leonore: Op. cit.

24. Matud, M. Pilar; GutiérReZ, Ana B. y Padilla, Vanessa: Op. cit.

25. LUNDY, M. y GROSSMAN, S.: "Clinical research and practice with battered women. What we know, what we need to know», Trauma, Violence \& Abuse, 2 (2001), pp. 120-141.

26. ECheburua, Enrique; Corral, Paz; SARAsúa, Belén y ZubizARRETA, Irene: «Mujeres víctimas de maltrato", en E. Echeburua y P. Corral (eds.): Manual de violencia familiar, Madrid, S. XXI, 1998, pp. 11-73. 
Algunas claves para una psicoterapia de orientación feminista en mujeres que...

Por otra parte, recientemente, Labrador, Paz, De Luis y Fernández R. ${ }^{27}$ han propuesto dos programas complementarios de intervención, para mujeres víctimas de violencia en la pareja: el programa de acción inmediata y el programa de tratamiento.

El Programa de Acción inmediata ha sido desarrollado por Pilar de Luis en el Servicio de Atención a Víctimas (SAV) de Delitos Violentos del Decanato de los Juzgados de Madrid y está pensado para actuar en situaciones de crisis y atender a las mujeres cuando acuden a este servicio, que suele ser tras haber denunciado su situación. Este programa se asemeja al implementado por el Colegio Oficial de Psicológos de Balears, con el patrocinio del Institut Balear de la Dona, cuyos óptimos resultados conocemos, aunque no se han publicado todavía.

Este programa se realiza habitualmente en 2 ó 3 sesiones de unas 2 horas de duración y consta de las siguientes fases: a) La fase de expresión emocional tiene como objetivo acoger a la víctima y facilitarle la expresión y comprensión de su situación de maltrato; b) La fase de valoración de la peligrosidad, tiene como objetivo evaluar el nivel de riesgo de que se produzca una nueva agresión y adoptar medidas para proteger a las mujeres; c) La fase de implementación de estrategias urgentes de afrontamiento, tiene como objetivo facilitar la toma de decisiones de la víctima e informar de las distintas opciones disponibles; d) La fase de evaluación psicológica, tiene como objetivo proporcionar una intervención terapéutica adecuada; y e) La fase de diseño de un plan de seguridad individualizado, tiene como objetivo incrementar la seguridad de la víctima.

El Programa de Tratamiento ha sido desarrollado por Labrador, F. J. y Rincón P. de la universidad Complutense de Madrid para reducir la sintomatología de estrés postraumático, tan habitual en las mujeres que han sido víctimas de esta forma de violencia.

Se trata de un programa grupal, pensado para ser aplicado en grupos con un número ideal de 4 participantes, con una periodicidad de una sesión semanal y una duración de 90 minutos por sesión. Está compuesto por una primera sesión en la que se aborda la psicoeducación acerca del problema, los objetivos del tratamiento y el concepto de violencia y el entrenamiento en relajación. Además, consta de 5 módulos: a) autoestima (2 sesiones), b) Estado de ánimo (2 sesiones), c) exposición (3 sesiones), d) Reevaluación cognitiva (2 sesiones) y e) Solución de problemas (2 sesiones). La selección de los módulos a aplicar y el orden de presentación de éstos dependerá de los síntomas (y de la gravedad de los mismos) que presenten las mujeres con las que se interviene en cada caso.

En nuestra opinión estos programas de tratamiento constituyen un ejemplo de la aplicación de la psicoterapia tradicional (en este caso de la terapia de raíz cognitivo-conductual) al problema de la violencia contra las mujeres en la pareja. Esto es, se toman los principios técnicos ya conocidos y se aplican, eso sí,

27. Labrador, Francisco J.; De Luis, Paulina; Pilar y Fernandez, Rocío: Mujeres víctimas de la violencia doméstica. Programa de actuación, Madrid, Pirámide, 2004. 
de una forma impecable, a un nuevo problema, pero sin introducir los matices derivados de las propias características de ese problema y sin contemplar la perspectiva de género y feminista que venimos reclamando como imprescindible en el abordaje de una temática como ésta.

También Matad, M.P. y cols. ${ }^{28}$ han diseñado un programa de tratamiento para mujeres que han sido víctimas de violencia en la pareja. Estas autoras sí consideran las particulares características de este problema, y, en base a ello, proponen un acercamiento integral que tenga en cuenta el contexto sociocultural y los factores que median la respuesta de la mujer ante el abuso y que incluye tanto técnicas cognitivo conductuales como otras generadas desde la perspectiva socio-estructural de análisis del maltrato.

La propuesta de intervención de estas autoras se centra en desarrollar las potencialidades de las mujeres y tiene como meta final ayudarlas a que recuperen el control sobre sus vidas y darles estrategias que las sitúen en una posición de mayor poder y confianza en sí mismas. Así, los objetivos específicos que se plantean en este programa son los siguientes: aumentar la seguridad de las mujeres; reducir y/o eliminar los síntomas que presenten; aumentar la autoestima y seguridad en sí mismas; aprender y/o mejorar sus estilos de afrontamiento, solución de problemas y toma de decisiones; fomentar la comunicación y unas habilidades sociales adecuadas; $y$ modificar sus creencias tradicionales sobre los roles de género y las actitudes sexistas.

Para todo ello sugieren emplear técnicas psicológicas como estrategias para el control de la ansiedad (relajación, respiración...), técnicas cognitivas para identificar y modificar pensamientos distorsionados (reestructuración cognitiva, parada de pensamiento...), inoculación de estrés. A todo ello se añade un componente educativo para abordar y modificar, en su caso, las creencias tradicionales sobre la violencia y las actitudes sexistas.

Las autoras insisten en la importancia de ajustar en cada caso la intervención a la situación concreta y a las necesidades de las mujeres. Además, sugieren que, dependiendo de las circunstancias, la intervención se puede realizar de forma individual, grupal o combinando ambos acercamientos.

Por el momento han evaluado los efectos de esta intervención sólo en un grupo reducido de mujeres (12), pero ha mostrado buenos resultados con reducciones significativas del estrés postraumático, la indefensión y la sintomatología depresiva ${ }^{29}$.

Aún valorando positivamente la aportación de Matud y cols., nuestra propuesta iría más en la línea propuesta por Leonore Walker ${ }^{30}$. Esta autora consideró que, definitivamente, la psicoterapia tradicional no es adecuada para aplicarla a mujeres que han sido víctimas de violencia de género, siendo necesario introducir modificaciones $y$, además, tener en cuenta tanto el impacto

28. Matud, M. Pilar; Gutiérrez, Ana B. y Padilla, Vanessa: Op. cit.

29. MATUd, M. PILAR: "Impacto de la violencia doméstica en la salud de la mujer maltratada», Psicothema, 16 (2004), pp. 397-401.

30. WALKER, Leonore E.A.: Op. cit. 
Algunas claves para una psicoterapia de orientación feminista en mujeres que...

específico del trauma que supone esta forma de violencia como la idiosincrasia concreta de la mujer a la que vaya a tratarse en cada caso.

Partiendo de estas premisas, esta autora compiló una seria de estrategias de intervención que tenían sus orígenes en la teoría feminista y en la terapia del trauma y que ella consideró que formaban una nueva intervención a la que denominó Survivor Therapy. Los principios que deben regir esta terapia serían, según esta autora, lograr que las mujeres que han padecido violencia logren alcanzar: su seguridad; su empoderamiento; validar sus experiencias; enfatizar sus puntos fuertes; diversificar sus alternativas; comprender la opresión que han padecido; tomar sus propias decisiones y juzgar con libertad los acontecimientos que viven.

\section{CONCLUSIONES}

En definitiva, a la vista de todo lo comentado, en nuestra opinión, la terapia a aplicar en las mujeres que han padecido violencia de género y, particularmente, violencia por parte de sus parejas, deberá asumir como principios fundamentales los siguientes: a) Ayudar a la toma de conciencia de la situación vivida, a la comprensión de que se ha sido o se está siendo víctima de un delito, ayudando a desentrañar los mitos sobre el amor y el matrimonio; b) Trabajar para la recuperación de los déficits psicológicos que puedan presentar las mujeres, y, especialmente de su autoestima.; c) Lograr la autonomía e independencia de las mujeres frente a la figura masculina; d) Ayudar a analizar la relación con los hijos, muchas veces mediatizada por la situación de violencia; e) Trabajar par la interiorización de los roles igualitarios. Nuevamente aquí será imprescindible deshacer mitos; f) Favorecer la reconstrucción de la vida social de la mujer; y en conclusión g) lograr el empoderamiento de las mujeres como objetivo final y paso hacia una nueva vida.

Pero, como ya hemos comentado y como recogen las palabras de Ángeles Alvárez "... si la asistencia no se articula con una política feminista que incluya un abanico de proyectos y propuestas claramente orientadas a eliminar la opresión de género, se corre el riesgo de diluirse en el asistencialismo que sólo puede, en el mejor de los casos, ayudar a algunas mujeres a limitar la violencia de sus vidas» ${ }^{31}$.

De hecho, y en términos más generales, deberíamos aceptar que, sin cambios sociales profundos, no podrá haber mejoras reales en la convivencia entre hombres y mujeres que propicien unas relaciones dignas y libres de violencia.

No obstante, somos conscientes de que esta premisa asusta a quienes se sienten cómodos en una sociedad que sigue dando por buenos y defendiendo, con más o menos virulencia, los privilegios en función del género, y, por tanto, están acomodados en unas relaciones asimétricas, en las que cada cual tiene muy claro cuál es su lugar, quién manda y quién debe obedecer.

31. Alvárez, Ángeles: Guía para mujeres maltratadas, Madrid, Consejo de la Mujer de la Comunidad de Madrid, 2001, p. 97. 
De esta manera, aunque en el mundo occidental ya casi nadie se atreva a cuestionar teóricamente los derechos de las mujeres, a lo hora de la verdad las resistencias son muchas. Es decir, frente a la ya poco discutida igualdad legal, existe todavía una más que dudosa igualdad real. Las alarmantes cifras sobre violencia de género lo demuestran, poniendo de manifiesto la existencia de un trasfondo oscuro y tenebroso en muchas relaciones afectivas, en las que se utilizan el acoso, la vejación, el insulto, los golpes, las amenazas y todo cuanto sea necesario para mantener el estatus «tradicional» y una situación de control y sometimiento femenino.

Las vidas perdidas, el sufrimiento generado, la desesperación, la frustración acumulada, las inteligencias ahogadas y desaprovechadas, los hijos e hijas infelices y desconcertados. Nada de todo esto es ya tolerable, ni la humanidad, aunque solo fuera por una visión egoísta de supervivencia, se lo puede permitir. El cambio es ya inaplazable.

Durante décadas la psicología y la psiquiatría se han mantenido al margen de todos estos planteamientos. Mas aún, en muchos casos, de una manera tanto explícita como implícita, han reforzado los supuestos sobre la existencia de diferencias «naturales» entre hombres y mujeres y su atribución a características internas propias de unos y otras, utilizando binomios como «masculinidad/feminidad», "pasividad/actividad» "emocionabilidad/racionalidad», que venían a confirmar el destino final de los seres humanos según nacieran hombres o mujeres en el mundo público, en que el se toman las decisiones (ellos) y en el privado y doméstico (ellas).

La incorporación de la perspectiva de género y, el surgimiento de una psicología de orientación feminista ha modificado este rumbo por lo que se refiere a la comprensión del comportamiento de las personas y la influencia que sobre él tiene la variable género. Es imprescindible que estas nuevas perspectivas lleguen también al ámbito de la psicoterapia, particularmente cuando ésta afronta problemas tan sensibles a estas cuestiones como aquellos que tienen que ver con la violencia de género y sus consecuencias. 